\title{
Observatoires et Infrastructures de Données Géographiques (IDG) pour le suivi spatiotemporel et environnemental des territoires.
}

\author{
Spatial Data Infrastructures (SDI) and observatories for the
}

spatiotemporal and the environmental monitoring.

\author{
Eric Masson ${ }^{1}$, Olivier Blanpain ${ }^{1}$ \\ ${ }^{1}$ Laboratoire Territoires Villes Environnement Société, Université de Lille, Avenue Paul Langevin, F-59650 Villeneuve- \\ d'Ascq, France
}

RÉSUMÉ. Cet article propose une réflexion sur les relations entre les observatoires, les infrastructures de données géographiques (IDG) et les besoins des utilisateurs pour le suivi spatiotemporel et environnemental des territoires. Après un rappel de la nature et des objectifs des observatoires et des IDG, nous analysons, à partir d'études de cas, différents systèmes d'observation, la mise à disposition des données géographiques collectées et l'adéquation de ces infrastructures de données avec les besoins et moyens des usagers. Nous nous appuierons ainsi sur le cas de la collecte d'imagerie multispectrale (satellitaire et aérienne) et de la donnée hydrométrique (mesures de débits en rivière) pour illustrer quelques-uns des défis numériques auxquels les observatoires doivent faire face pour atteindre leurs objectifs.

ABSTRACT. This paper proposes a reflection on the relations between the observatories, the Spatial Data Infrastructures (SDI) and the user needs for the spatiotemporal and the environmental monitoring. After a reminder of the nature and objectives of the observatories and the SDIs, we analyze from case studies different observation systems, the availability of collected geographical data using various SDI and the adequacy of these infrastructures with the needs and means of the users. We will rely on multispectral (satellite and aerial) imagery collection and hydrometric data collection (streamflow measurements) to illustrate some of the numerical challenges observatories face in achieving their goals.

MOTS-CLÉS. Observatoires, Infrastructures de Données Géographiques, Télédétection, Imagerie satellitaire, Sentinel, Imagerie aérienne, Hydrométrie, Risques hydrologiques.

KEYWORDS. Observatories, Spatial Data Infrastructures, Remote sensing, Satellite imagery, Sentinel, Aerial imagery, Hydrometry, Hydrological risks.

\section{Introduction}

Cet article est une contribution à la réflexion sur les usages des données scientifiques ouvertes de la recherche dans le cadre pluridisciplinaire de la télédétection et de l'hydrologie. Ces dernières décennies, le développement de nouvelles technologies et ressources numériques pour les capteurs (observation et acquisition de données), pour le stockage (versement, renseignement et enregistrement numérique des données), pour la mise à disposition des données (open data et interopérabilité) et pour leur analyse numérique (logiciels et tutoriels en libre accès) modifient profondément les habitus disciplinaires et ouvrent de nouvelles perspectives scientifiques en parallèle de nouveaux défi méthodologiques. Les Infrastructures de Données Géographiques (IDG) sont un exemple de ces nouvelles ressources très récentes, au regard des questionnements disciplinaires d'observation et de suivi environnemental de la surface terrestre ou de l'hydrologie continentale. Cette (r)évolution tant dans l'accès que dans le volume des données numériques disponibles (open big data) ouvrent à de nouvelles pratiques dont l'interopérabilité est un élément clef (Percivall, 2010). Nous considérons aussi une IDG comme un résultat de la recherche (instrumentation en hydrométrie, en imagerie...), car le producteur de données ne cesse de faire progresser ces capacités techniques d'observation pour la mise à disposition de nouvelles données pour la recherche. Nous considérons aussi les IDG comme des observatoires pour et par la 
recherche, car l'utilisateur final, scientifique ou institutionnel, y collecte les données nécessaires à l'observation et à l'analyse de son territoire. Les vocations des IDG sont principalement la gestion et l'accès par l'interopérabilité de données géolocalisées pour alimenter des modèles numériques de traitements finalisés, disciplinaires ou pluridisciplinaires. Il existe de ce fait une forte interdépendance entre les observatoires, les IDG, la recherche et les besoins des utilisateurs pour le suivi spatiotemporel et environnemental des territoires (Georis-Creuseveau, 2014; GeorisCreuseveau et al., 2015, Noucher, 2016 ; Noucher et al., 2017 ; Raj Budhathoki 2008 ; da Silva E. S. et Camboim S. P., 2018).

Notre expérience de recherche sur le risque inondation et sur le suivi des états de surfaces à l'aide de différentes sources d'imagerie aérienne et satellitaire nous a confronté à la dispersion des sources de données géographiques et hydrologiques ainsi qu'aux limites du formatage de leurs résolutions numériques, spatiotemporelles et de leur mise à disposition. Après un rappel de la nature et des objectifs des observatoires, nous analysons, à partir d'études de cas, différents systèmes d'observation, la mise à disposition des données géographiques qu'ils produisent et l'adéquation de ces infrastructures avec les besoins et moyens des usagers. Nous nous appuierons ainsi sur la collecte d'imagerie optique (satellitaire et aérienne) et radar (satellitaire) et la collecte de données hydrométriques (mesures de débits en rivière) pour illustrer quelques-uns des défis numériques auxquels les observatoires doivent faire face pour atteindre leurs objectifs de suivi territorial en croisant des données spatiotemporelles issues de l'imagerie et des données temporelles issues de mesures ponctuelles.

\section{Observatoires et observations environnementale des territoires}

Les observatoires territoriaux ou environnementaux répondent à une demande croisée des acteurs institutionnels, des populations et des scientifiques. Les OHM (Observatoire Hommes-Milieux) du labex DRIIHM (http://www.driihm.fr/), les programmes d'observation Copernicus de l'Agence Spatiale Européenne (ESA) Earth Resources Observation and Science (EROS) l'United State Geological Survey (USGS) sont quelques exemples d'observatoires dont les missions s'appuient sur et par la mise à dispositions de données environnementales à références spatiales. Dans ce contexte nous proposons d'analyser le lien entre «observatoires » et « observations » à travers l'exemple de l'imagerie aérienne et satellitaire et celui de l'hydrométrie. Ces deux types de données sont complémentaires sur la thématique du risque inondation et elles sont, à ce titre, conjointement mobilisées par les chercheurs et les acteurs des territoires. Pourtant, ces données relèvent de protocoles d'acquisition et de suivi des dynamiques spatio-temporelles très différents.

\subsection{Observatoires par imagerie aérienne et satellitaire}

L'imagerie aérienne ou satellitaire est une donnée produite principalement par des observatoires institutionnels (agences spatiales, Institut Géographique National ou équivalent). En France, la photographie aérienne est utilisée de manière systématique pour cartographier l'occupation du sol dès les années 35 à 39 (Chevalier, 1963) par le service géographie de l'armée puis par l'Institut Géographique National (IGN) à partir de 1940. Les photographies utilisées sont tout d'abord de type panchromatique, puis couleur à la fin des années 80 (figure 1). Cette imagerie a pour objectif de fournir la connaissance du terrain et de son occupation du sol qui est nécessaire à la réalisation de la couverture nationale de cartes topographiques à $1 / 50000$ et $1 / 25000$ jusque dans les années 90 . La couverture systématique de la France par photographie aérienne est mise en place progressivement, devenant ainsi un observatoire territorial national. Dans les années 90, l'imagerie aérienne par caméra numérique se substitue aux clichés réalisés par photographie argentique, historiquement utilisée par l'IGN (Souchon et al., 2006, Touzet et Lecordix, 2010). La première couverture numérique et systématique de l'orthophotographie nationale (BD Ortho®) est disponible pour l'année 2003 et elle intègre en 2008 le Référentiel à Grande Echelle (RGE). La mise à jour de la BDOrtho ${ }^{\circledR}$ est actuellement de quelques années seulement. En France, d'autres observatoires 
territoriaux par imagerie sont également déployés dans la deuxième moitié du $20^{\mathrm{e}}$ siècle. Ainsi, à la fin des années 60, l'Inventaire Forestier National (IFN) utilise l'imagerie aérienne infrarouge avec une période de revisite d'environ 10 ans pour réaliser la cartographie nationale et la gestion des ressources forestières (Touzet et Lecordix, 2010). L'imagerie aérienne de l'IGN et de l'IFN répond donc aux besoins d'observation systémique du territoire. En 1986, la mission SPOT (Satellite d'Observation Probatoire de la Terre) du Centre National d'Etudes Spatiales (CNES) étend la logique de l'observatoire par imagerie optique systématique à l'échelle globale. Là encore les progrès technologiques (capteurs numériques, télécommunication, stockage numérique, puissance de calcul...), accompagnent l'évolution des modalités d'acquisition et des applications de l'imagerie satellitaire.

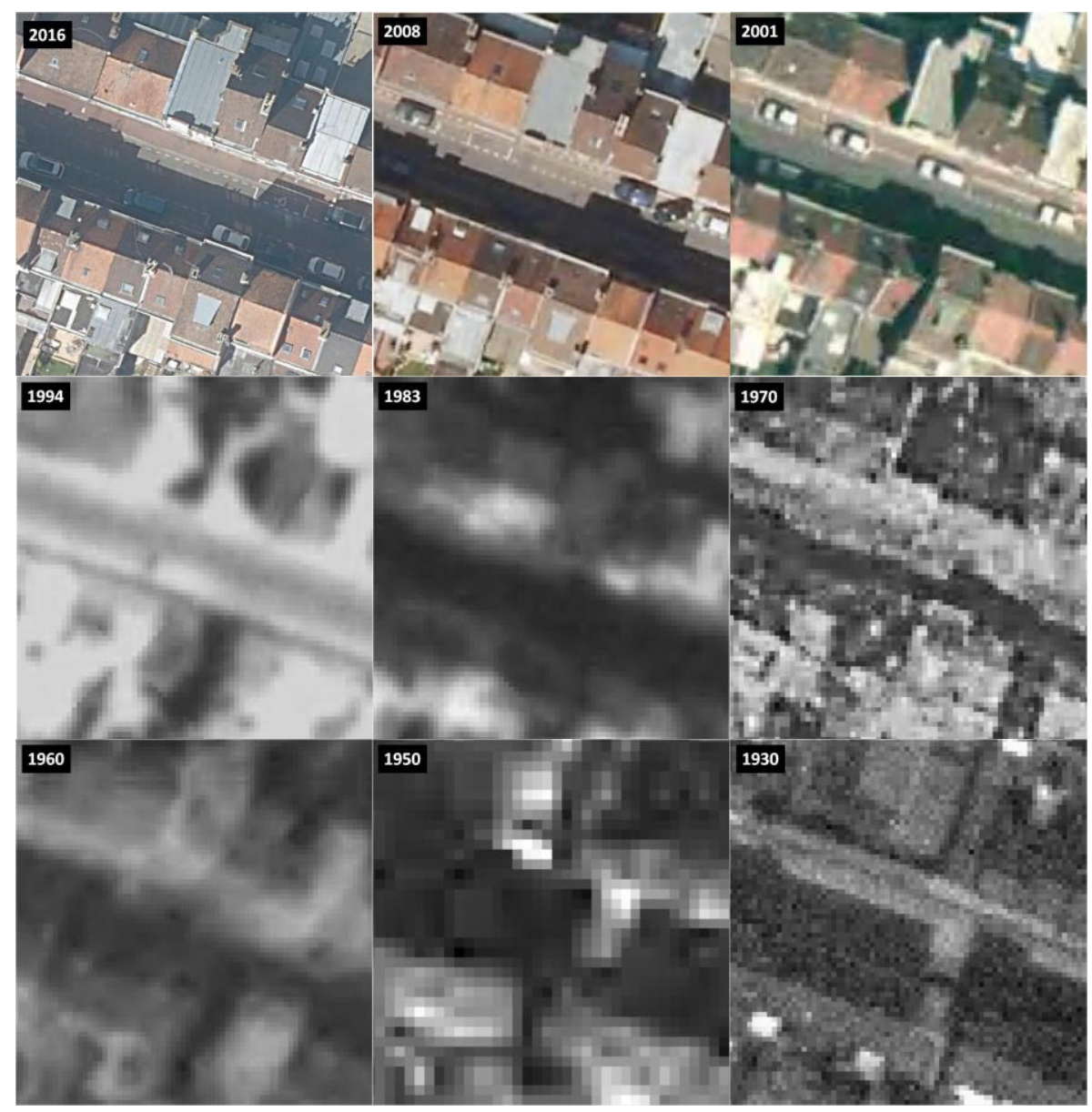

Figure 1. Evolution des techniques d'acquisition aérienne : $8 \mathrm{~cm}$ de résolution spatiale en 2016 contre $1 \mathrm{~m}$ en 1950 (source : MelMap 2019, http://carte.melmap.fr/)

Les images aériennes et satellitaires sont des données à références spatiales qui proposent un échantillonnage de l'état de surface au sol dans le domaine du rayonnement électromagnétique. Par rapport à l'imagerie aérienne, l'avantage de l'observatoire satellitaire de type SPOT ou Landsat correspond à une plus grande répétitivité temporelle (quasi mensuelle), une plus grande couverture géographique et une meilleure résolution spectrale. En contrepartie, la précision géométrique décroit avec une dégradation de la résolution spatiale qui devient décamétrique et supérieure pour l'imagerie satellitaire de la fin du $20^{\mathrm{e}}$ siècle. La course à la résolution spatiale entre imagerie aérienne et satellitaire a aujourd'hui atteint un point ou l'échantillonnage par imagerie satellitaire est sub-métrique $(30 \mathrm{~cm}$ de résolution pour Worldview 3) alors que l'imagerie aérienne est centimétrique (8 cm de résolution l'orthophotographie 2016 sur la Métropole de Lille, figure 1). Il existe d'autres types de données acquises par vecteur aéroporté ou satellitaire. Des données aériennes lidar (topographie capteur laser) sont aussi mises à disposition par l'IDG de l'observatoire de la mer et du littoral (http://www.geolittoral.developpement-durable.gouv.fr/). Les données satellitaires radar du programme Sentinel sont mises à disposition par l'IDG du programme 
Copernicus. Toutes ces données d'observations s'accumulent dans les catalogues des producteurs/fournisseurs d'images numériques à références spatiales.

La contrainte de stockage et de mise à disposition des archives, de plus en plus nombreuses et volumineuses, pousse les observatoires à l'interopérabilité de leurs catalogues avec des IDG conçues pour faciliter l'extraction des données et en assurer la valorisation (Aditya et Kraak, 2007 ; Bernard et al, 2017 ; da Silva et Camboim, 2018 ; Devillers et al., 2007, Noucher, 2016 ; Noucher et Gautreau, 2013 ; Percivall, 2010 ; Raj Budhathoki et al., 2008 ; Wiemann et Bernard, 2016). Cette contrainte numérique est une conséquence des stratégies d'observation systématique (globale, nationale voir régionale), de la progression des résolutions (numériques, spectrales, spatiales et temporelles) et de l'accumulation des données archivées. En effet, l'évolution technologique de l'imagerie satellitaire et aéroportée se fait au prix d'une très forte inflation du poids numérique des fichiers d'archive. A titre d'exemple pour les données de la série Landsat, il était d'environ 100 Mo pour une archive MSS de 1972 et il atteint environ 1,8 Go pour une archive OLI de 2018. Landsat 8 OLI produit une imagerie globale de la planète tous les 16 jours et fait l'acquisition de l'état de surface terrestre d'environ 21 mio $\mathrm{km}^{2}$ par jour. Or la plupart des chercheurs préfère une donnée de qualité qui répond à leurs attentes spécifiques qu'une masse de données qui nécessite une recherche en plusieurs étapes (Willard et Berbert, 1999).

L'interopérabilité des formats de métadonnées entre différents production d'imagerie satellitaire permet à de multiples IDG d'accéder aux catalogues de différents fournisseurs (Noucher et al., 2017 ; Percivall, 2010 ; Vancauwenberghe et al., 2018. Wiemann et Bernard, 2016). La figure 2 présente un exemple d'interopérabilité entre l'IDG Earthexplorer de l'USGS et l'IDG Copernicus de l'ESA qui offre un accès open data à l'ensemble des données du programme Sentinel (figure 2). Ces observatoires sont des fournisseurs de données à références spatiales dont la clientèle reste principalement institutionnelle (Noucher et Gautreau, 2013 ; Georis-Creuseveau, 2014) mais aussi d'autres IDG. Leur usage, pour certaines applications et notamment le suivi, l'analyse et la cartographie des aléas hydrologiques, reste parfois problématique pour l'utilisateur final (Borah et al., 2018 ; Chung et al., 2015 ; Dao et Liou, 2015 ; Demir et Krajewski, 2013 ; Frazier et al., 2003 ; Hoque et al., 2011 ; Jain et al., 2005 ; Khan et al., 2011 ; Revilla-Romero et al., 2015 ; Sun et al., 2018 ; Wang et Xie, 2018). L'acquisition des données satellitaire, qui est basée sur des paramètres instrumentaux de capteurs numériques (optique, radar, résolutions...) et un protocole d'acquisition (héliosynchronisme, fauchée, période de revisite...), est aussi limitée par une sensibilité aux nuages (données optiques), à la topographie ou au vent de surface (données radar) et pour un horaire de revisite quasi fixe (tout type d'image). Ces contraintes ne sont pas en relation spatiotemporelle directe avec la dynamique physique des aléas hydrologiques. En d'autres termes, il n'existe pas d'IDG susceptible de fournir une donnée satellitaire, à une résolution opérationnelle de terrain, à n'importe quelle heure de la journée. La plupart des satellites optiques proposent une acquisition autour de 12:00 (heure solaire locale) à une latitude choisie qui correspond en général aux latitudes moyennes de l'hémisphère nord. Il s'agit ainsi de minimiser les effets induits par l'incidence solaire et le relief (ombres portées) sur les territoires prioritaires à l'observation. Cela permet aussi d'optimiser les paramètres orbitaux pour la quantification du rayonnement solaire réfléchi qui est l'observation physique réalisée par un capteur imageur numérique. 


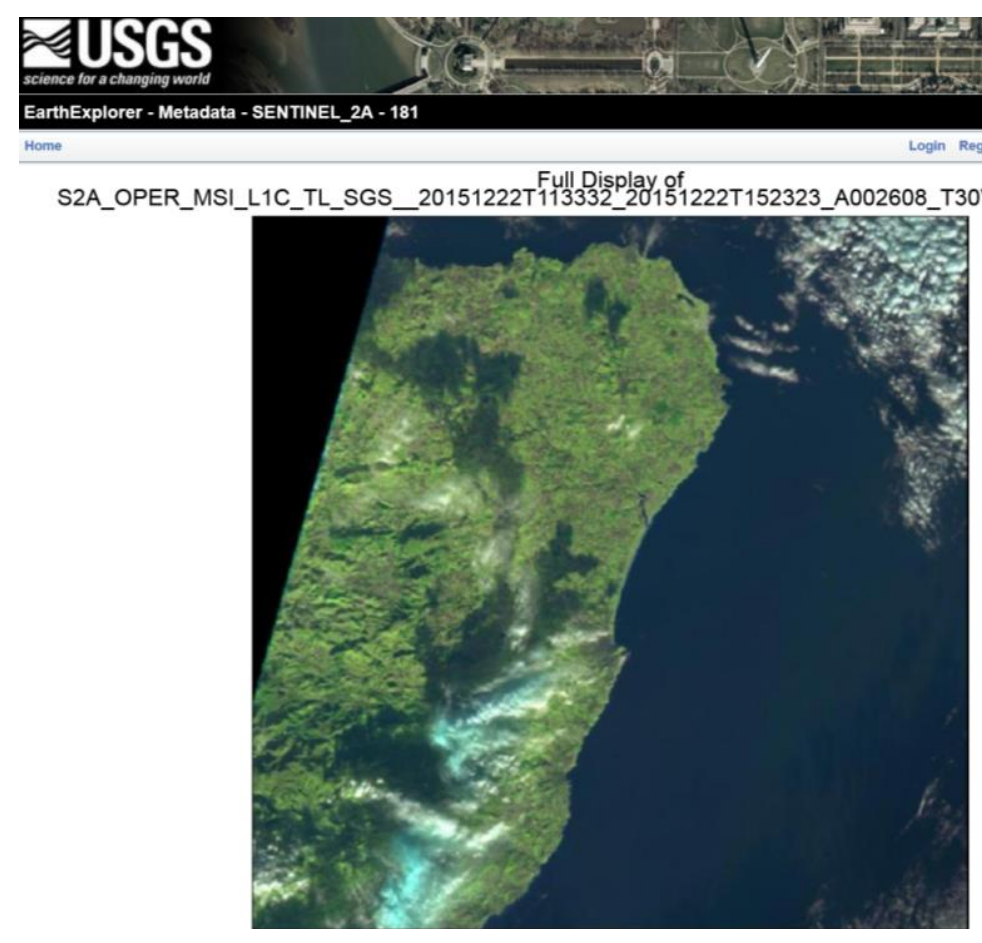

Figure 2. Visualisation de métadonnées qualitatives au format image pour l'extraction d'une archive Sentinel 2-A depuis l'IDG de I'USGS.

La finalité d'usage thématique, comme le suivi, l'analyse et la cartographie des aléas hydrologiques, reste parfois aléatoire pour l'utilisateur-acteur territorial. Les IDG satellitaires proposent une observation qui est souvent décalée par rapport au niveau maximum atteint par une inondation. Les programmations d'urgence offrent une plus grande adéquation aux besoins utilisateurs en dépassant certaines contraintes temporelles du protocole d'acquisition. Cependant les contraintes physiques de la mesure (optique ou radar) ne sont pas pour autant effacées avec le risque d'un résultat en deçà des attentes utilisateur pour un surcoût de programmation d'urgence qui limite fortement l'usage de ce type de solution à quelques institutions transnationales (UNOSAT).

La cartographie de la zone maximale d'extension d'une inondation suppose une prévision temporelle des niveaux d'eaux (Bormann et al., 2011 ; Chung et al., 2015 ; Frazier et al., 2003, Gu et al., 2016, Khan et al. 2011, Knebl et al. 2005 ; Kundzewicz et al., 2005 ; Merz et al., 2016, 2018 ; Nied et al., 2014 ; Revilla-Romero et al., 2015) qui soit compatible avec les paramètres de programmation d'urgence pour l'acquisition de données satellitaires. Cependant, les paramètres atmosphériques peuvent empêcher une acquisition optique directe à très haute résolution spatiale en cas de couverture nuageuse ou de pic de crue pendant la nuit. L'emploi d'une programmation par imagerie satellitaire radar, insensible aux nuages et à l'absence de lumière naturelle, est une solution qui permet de pallier les déficiences des capteurs optiques au prix d'une réduction de la résolution spatiale dans des conditions favorables de vent de surface, de topographie et d'angle d'acquisition.

S'agissant de la programmation d'acquisition de données aériennes, il faut pouvoir organiser dans l'urgence un plan de vol et une acquisition. Ce type de couverture est aussi affecté aussi par un décalage temporel entre les images et l'extension maximale de l'inondation. Dans les deux cas, acquisition satellitaire ou aérienne, il très difficile d'observer le niveau maximum atteint par une inondation car celui-ci se propage de l'amont vers l'aval de la vallée avec des temps de propagation qui sont variables d'une inondation à l'autre et d'un cours d'eau à l'autre. Il faudrait une imagerie continue pour pouvoir capturer la dynamique hydrologique et extraire la courbe enveloppe du maximum d'inondation. Dans tous les cas, les événements de très grande emprise géographique imposent des limites techniques et une contrainte budgétaire sur l'acquisition par programmation qui renvoient l'utilisateur final vers l'imagerie satellitaire d'archive pour mener à bien son observation à posteriori. Accéder plusieurs sources d'acquisition satellitaire ou plusieurs IDG et la seule solution 
pour pallier les lacunes d'une seule source ou d'un seul IDG. En effet, la variabilité des protocoles d'acquisition et des paramètres orbitaux des satellites EO (Earth Observation) offre une complémentarité qui est souvent recherche par l'utilisateur qui ne trouve pas la donnée nécessaire dans le silo de référence de son IDG habituelle.

\subsection{Observatoires hydrologiques par mesure ponctuelle}

En France, le suivi hydrologique des cours d'eau pour l'alerte (déploiement des services d'annonces des crues en France à la fin du $19^{\mathrm{e}}$ siècle) puis la prévision des crues au début du $21^{\mathrm{e}}$ siècle (création du SCHAPI et du service vigicrues https://www.vigicrues.gouv.fr/) a accompagné l'évolution technologique des outils de mesure hydrométrique, de la télétransmission des données numériques, puis de la géovisualisation en ligne des observations hydrologiques en quasi-temps réel. Comme dans beaucoup d'autres pays, il existe donc en France un observatoire national de l'hydrométrie des cours d'eau. L'acquisition numérique des données hydrométriques se fait à haute fréquence temporelle depuis la fin des années 90 . Cette acquisition est paramétrable à distance et peut varier de quelques minutes à quelques dizaines de minutes entre chaque mesure, contre une à trois observations journalières au début $\mathrm{du} 20^{\mathrm{e}}$ siècle (Masson, 2002; Kharroubi O., 2013 ; Kharroubi et al. 2016). En comparaison de la fréquence d'acquisition des données issues de l'imagerie, cette mesure à haute fréquence permet de bien échantillonner le caractère très dynamique des écoulements, notamment en période de crue ou de décrue. Cette haute fréquence d'acquisition s'explique par l'évolution technologique de l'hydrométrie sur les 100 dernières années mais aussi parce qu'il s'agit d'une mesure ponctuelle dont le coût numérique de transmission et de stockage est minime pour chaque observation et reste très faible même pour une très longue chronique hydrologique.

L'observation hydrométrique a ses incertitudes, qui ne seront pas discutées ici, mais elle permet d'établir un état des écoulements à l'amont du point de mesure dans le lit mineur ou dans le lit majeur d'un cours d'eau. Il s'agit donc d'une valeur d'intégration spatiotemporelle des processus de drainage d'un territoire hydrologique : le bassin versant. Cette mesure hydrométrique est très précise en hauteur ce qui permet de définir des lignes d'eau qui sont très utiles pour modéliser ou cartographier les limites des zones inondées et la durée de submersion. La mesure des hauteurs d'eau est donc très fiable, quasi millimétrique aujourd'hui, centimétrique au début du $20^{\mathrm{e}}$ siècle. Par contre, cette observation ponctuelle ne présente pas une couverture spatiale étendue car elle se limite à une ligne d'eau en travers de la vallée. C'est ce qui justifie l'approche combinée entre la mesure hydrométrique et l'imagerie aérienne ou satellitaire en période de crue. Une autre limite à l'usage de cette donnée concerne la stationnarité de la mesure. Déplacer une station, même de quelques mètres c'est introduire un biais d'observation et une rupture dans la fiabilité des chroniques hydrométriques. Cela impose de corriger les données pour pouvoir les comparer avec les chroniques plus anciennes ou d'autres stations. L'évolution de l'occupation du sol, les événements érosifs de crues ou décrues peuvent également modifier très fortement ces conditions de stationnarité (Wang et $a l ., 2015)$. Dans la pire des situations hydrométriques, qui correspond à une incapacité à corriger l'étalonnage de la mesure, le site choisi n'est pas stable (la données n'est alors plus fiable). Il peut aussi être détruit par un événement hydrologique extrême ; l'observation n'est alors plus possible et une lacune temporelle s'installe dans la chronique hydrométrique. L'usage des données hydrométriques s'accompagnent donc de métadonnées qui précisent en plus du positionnement géographique, la qualité et la quantité d'information disponible dans une chronique. Ces métadonnées sont utilisées pour structurer et alimenter les IDG en charge de diffuser les observations hydrométriques.

Les observatoires par imagerie ou par mesure hydrométrique ponctuelle sont complémentaires pour l'alerte, l'analyse et la préparation et la gestion de crise hydrologique. Les services de l'Etat, d'intervention et de secours, les acteurs du territoire ou les spécialistes du risque recherchent donc le plus souvent à croiser ces deux sources de données pour produire une observation spatiotemporelle 
restituant au mieux la dynamique hydrologique d'un aléa. Cette information est nécessaire pour limiter les conséquences des crues et porter secours le plus efficacement possible auprès des populations concernées. Dans les vallées des très grands fleuves de la planète, la mise en œuvre de l'aide d'urgence ne peut se faire sans une cartographie par des données satellitaires de l'accessibilité des lieux d'habitations inondés (UNOSAT, 2017).

\section{Des IDG pour la mise à disposition des données}

«La généralisation de la production de données géographiques, l'accélération de leurs flux et l'expansion de leurs usages engendrent aujourd'hui une démultiplication de l'offre disponible sur le web. Cette situation ... génère de nouveaux enjeux de recherche, ... pour ... rendre visibles les contenus ... diffusés par des infrastructures de données souvent opaques ... un enjeu sociétal ... acteurs publics et privés ... s'interrogent sur l'impact de ces nouveaux dispositifs en termes de gouvernance et de gestion territoriale. » (Manifesto du site GEOBS - http://www.geobs.cnrs.fr).

\subsection{IDG et suivi spatiotemporel des états de surfaces}

L'accès aux données géospatiales passe nécessairement par des IDG sur le web. Ces IDG respectent pour la plupart, sinon pour les plus récentes, les formats interopérables de l'OGC (Desconnets, 2017, Haller et al., 2019, vand den Brink et al., 2019) ce qui permet à l'utilisateur d'interroger les catalogues en s'appuyant sur les informations contenues dans les métadonnées (Aditya, 2007 ; Bernard, 2017 ; Chevalier et Caron, 2002). Celles-ci, le plus souvent au format text ou XML (Desconnets, 2017), permettent aussi à d'autres IDG d'échanger des informations sur les catalogues et, pour les principaux observatoires institutionnels, d'accéder directement aux flux de données (API Hub du programme Copernicus: https://scihub.copernicus.eu/) ou à leur téléchargement après identification de l'usager (Earthexplorer de l'USGS : https://earthexplorer.usgs.gov/). S'agissant de l'imagerie aérienne ou satellitaire, les catalogues de données sont organisés en silos et chaque entrée correspond à une archive composée de plusieurs fichiers de données et de métadonnées (Smith, 1996 ; Aditya et Kraak, 2007). L'enregistrement d'une image aérienne ou satellitaire dépend des paramètres d'observations définis par la stratégie de suivi spatiotemporel des états de surface, elle-même définie par les objectifs poursuivis par l'observatoire. Dans le domaine de l'imagerie, les résolutions spectrales, spatiales, temporelles et numériques varient suffisamment d'un capteur à l'autre pour éviter la redondance ou la concurrence directe entre plusieurs systèmes d'observation (tableau $\mathrm{n}^{\circ} 1$ ).

Les principales agences spatiales partagent également la volonté d'assurer une continuité des missions d'observation systématique de la surface terrestre malgré les évolutions technologiques depuis les années 70. Parmi ces missions d'observation, les programmes Landsat 1 à 8 de la NASA (débutant en 1972), Spot 1 à 7 du CNES (débutant en 1986) et Jers-1/Palsar de la JAXA (débutant en 1992) sont trois exemples dans le domaine de la télédétection optique et radar où la continuité des missions d'observation, que nous assimilons à des observatoires, a été préservée tout en bénéficiant des avancées technologiques des systèmes et capteurs d'acquisition. 


\begin{tabular}{|c|c|c|c|}
\hline & Landsat 8 & Sentinel 2A-2B & Spot 6-7 \\
\hline Fauchée (km) & 185 & 290 & 60 à 180 \\
\hline $\begin{array}{l}\text { Résolution spectrale } \\
\text { (bandes et longueurs } \\
\text { d'onde en } \mu \mathrm{m} \text { ) }\end{array}$ & \begin{tabular}{|} 
Pan : 0,500-0,680 \\
Aérosols : $0,433-0,453$ \\
Bleu : $0,450-0,515$ \\
Vert : $0,525-0,600$ \\
Rouge : $0,630-0,680$ \\
PIR : $0,845-0,885$ \\
Cirrus : $1,360-1,390$ \\
IRC $1: 1,560-1,660$ \\
IRC $2: 2,100-2,300$ \\
IRT $1: 10,30-11,30$ \\
IRT $2: 11,50-12,50$
\end{tabular} & $\begin{array}{l}\text { Aérosols : 0,43-0,457 } \\
\text { Bleu : 0,447-0,545 } \\
\text { Vert : 0,537-0,582 } \\
\text { Rouge : 0,645-0,683 } \\
\text { RE } 1: 0,694-0,713 \\
\text { RE } 2: 0,731-0,749 \\
\text { PIR } 1: 0,762-0,907 \\
\text { PIR } 2: 0,768-0,796 \\
\text { PIR } 3: 0,848-0,881 \\
\text { Vapeur d'eau : 0,932-0,958 } \\
\text { Cirrus : } 1,336-1,41 \\
\text { IRC } 1: 1,542-1,685 \\
\text { IRC } 2: 2,081-2,323\end{array}$ & \begin{tabular}{|c} 
Pan : $0,450-0,745$ \\
Bleu : 0,450-0,520 \\
Vert : $0,530-0,590$ \\
Rouge : 0,625-0,695 \\
PIR : $0,760-0,890$
\end{tabular} \\
\hline $\begin{array}{l}\text { Résolution spatiale par } \\
\text { domaine spectral }\end{array}$ & $\begin{array}{c}15 \mathrm{~m} \text { (Pan), 30m (MS), } \\
100 \text { (IRT) }\end{array}$ & $\begin{array}{l}\text { 10m (MS\&PIR), 20m } \\
\text { (PIR\&IRC), 60m (Atm) }\end{array}$ & $\begin{array}{c}1,5 \mathrm{~m} \text { (Pan), } \\
3 \mathrm{~m} \text { (MS) }\end{array}$ \\
\hline Résolution numérique & 12 bits & 12 bits & 12 bits \\
\hline Résolution temporelle & $16 \mathrm{j}$ & $5 j$ & $3 \mathrm{j}$ \\
\hline Horaire passage équateur & 10h11 & $10 \mathrm{~h} 30$ & $10 \mathrm{~h}$ \\
\hline Capacité d'observation & $21 \mathrm{mio} \mathrm{km}^{2} / \mathrm{jour}$ & $12 \mathrm{mio} \mathrm{km}^{2} /$ jour (estimation) & $6 \mathrm{mio} \mathrm{km}^{2} / \mathrm{jour}$ \\
\hline \begin{tabular}{|l|}
$\begin{array}{l}\text { Domaines spectraux des } \\
\text { capteurs embarqués }\end{array}$ \\
\end{tabular} & $P a n+M S+I R T$ & $\mathrm{Pan}+\mathrm{MS}+\mathrm{IRC}$ & Pan + MS \\
\hline \multicolumn{4}{|c|}{$\begin{array}{l}\text { Abréviations : Pan (Panchromatique), MS (MultiSpectral), RE (RedEdge = sommet/pic du rouge), PIR } \\
\text { (Proche InfraRouque), IRC (InfraRouge ondes Courtes), IRT(InfraRouge Thermique), Atm (Qualité } \\
\text { Atmosphérique) } \\
\text { Références utilisées: } \\
\text { https://www.intelligence-airbusds.com/files/pmedia/public/r12784_9_spot6- } \\
\text { 7_fiche_technique.pdf } \\
\text { https://landsat.usgs.gov/sites/default/files/documents/Landsat8DataUsersHandbook.pdf } \\
\text { http://esamultimedia.esa.int/docs/GMES/Sentinel-2 MRD.pdf }\end{array}$} \\
\hline
\end{tabular}

Tableau 1. Comparaison de trois systèmes d'observation de la surface terrestre

Pour exploiter les catalogues d'imagerie, l'utilisateur dispose donc de systèmes de requêtes croisant une emprise spatiale (fournie par l'utilisateur) avec des attributs spatiotemporels et paramétriques fournis par les métadonnées (Smith, 1996 ; Aditya et Kraak, 2007). La procédure la plus classique d'extraction de données depuis une IDG fonctionne en quatre étapes (figure 3). Elle s'appuie sur le Modèle Logique de Données (MLD) de l'observation satellitaire pour définir la structuration de l'IDG et des catalogues de données qui y sont archivés. 


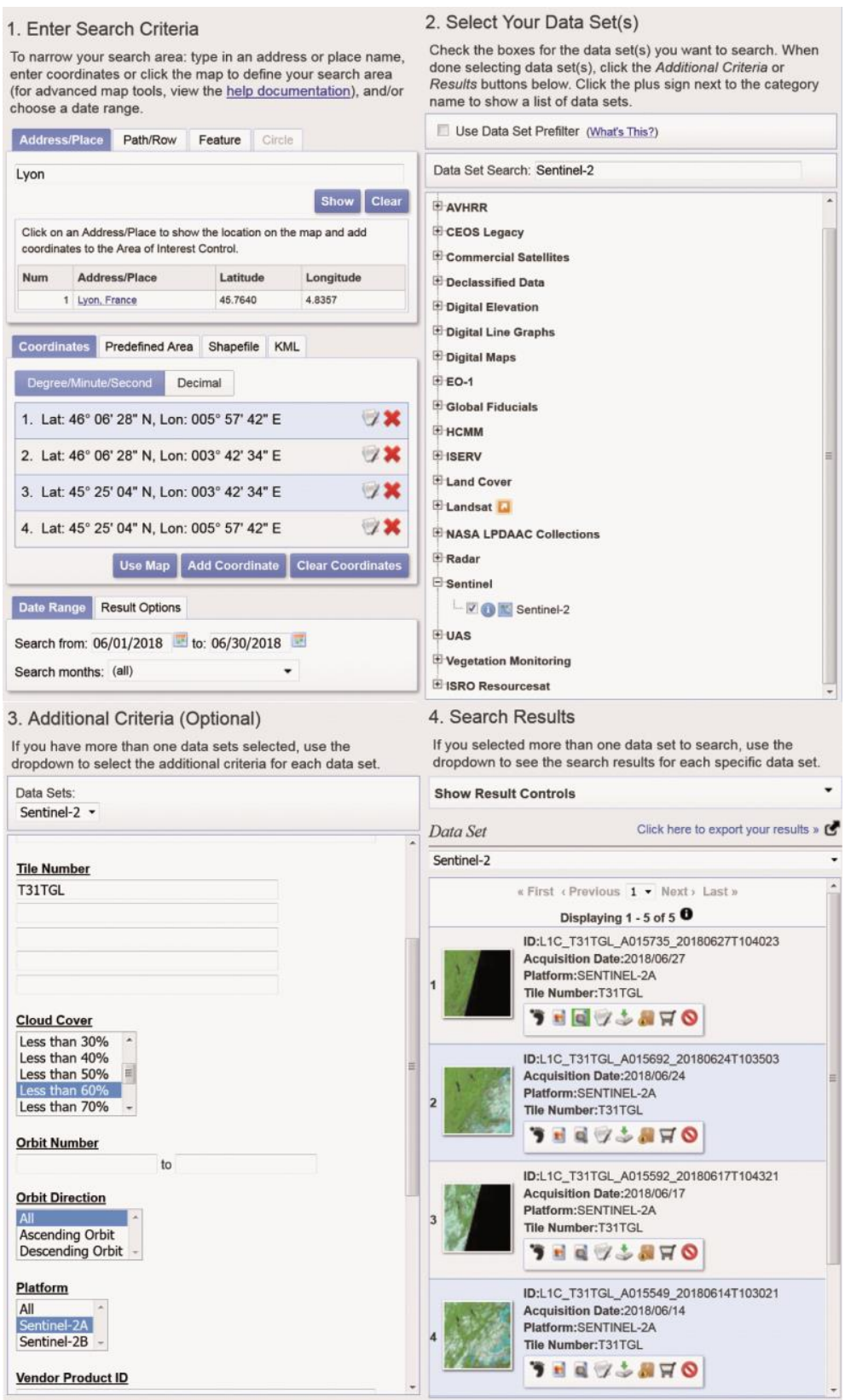

Figure 3. Procédure de requêtage en 4 étapes pour l'extraction de données Sentinel 2 depuis l'IDG Earthexplorer (https://earthexplorer.usgs.gov/).

Dans le cas de l'IDG Earthexplorer (figure 3) l'utilisateur définit l'emprise spatiale et le segment temporel pour l'extraction souhaitée. L'emprise spatiale peut être renseignée de plusieurs façons : par renseignement de toponyme, par jeux de coordonnées ponctuelles $(\mathrm{x}, \mathrm{y})$, par téléchargement d'un fichier SIG (au format interopérable *.shp, ${ }^{*} . \mathrm{kml} / \mathrm{kmz}$ ) ou par saisie manuelle d'un polygone d'emprise depuis la fenêtre de géovisualisation. Cette première étape de la requête spatiotemporelle vise donc à intersecter deux emprises spatiales (utilisateur et archives) pour une fenêtre temporelle définie par l'utilisateur. La deuxième étape consiste à sélectionner le catalogue de données dans lequel la requête spatiotemporelle doit être appliquée. La troisième étape permet à l'utilisateur d'intégrer d'autres critères issus de la lecture des métadonnées comme des paramètres instrumentaux (choix de l'orbite, des instruments actifs, capteurs dans le cas de plateforme multicapteurs, polarisation, angle de vue...), des paramètres d'archivage (identifiant catalogue, niveau de traitement de la donnée brute...). Pour les données optiques, l'indicateur de couverture nuageuse est également un critère d'extraction incontournable dans les systèmes de requête des IDG. 
Plusieurs IDG archivent et administrent l'accès aux données hydrométriques comme par exemple la Banque Hydro (BH) en France ou le National Water Information System (NWIS) aux USA. Si l'ouverture des données hydrologiques au public est très récente en France, elle existe depuis longtemps aux Etats-Unis et, depuis 2001, l'accès à ces données hydrologiques à références spatiales est possible depuis le site du NWIS (https://waterdata.usgs.gov/nwis). Les IDG NWIS et $\mathrm{BH}$ (http://www.hydro.eaufrance.fr/) proposent un accès à des données un peu différent (tableau 2).

Contrairement à la $\mathrm{BH}$ les outils de visualisation/extraction de données sont plus complets (figure 4) pour le NWIS et ils s'appuient sur une interface de type WebGIS avec un système de requêtage spatial (maillage administratif et hydrologique, emprise spatiale par polygone ou géolocalisation des stations de mesure) ou de requêtage attributaire (identifiant de la station hydrométrique, types de données hydrologiques et temporalités d'observation (Beran et Piasecki, 2009 ; Tarboton et al. 2009 ; Ames et al., 2012). La BH utilise des menus déroulants pour sectoriser la recherche des données à partir des métadonnées des fiches de stations qui recensent les informations de maillage territorial hydrologique (bassin versant, cours d'eau, station).

Si le NWIS donne un accès aux données brutes et à un rapport de synthèse hydrologique (tableau 2), la $\mathrm{BH}$ propose une série d'informations élaborées à partir de traitements automatiques des mesures hydrologiques collectées (données CRUCAL, VCX-QCX, VCN-QCN, QTFIX, QTVAR, et qualité de la mesure, tableau ${ }^{\circ} 2$ ). De son côté le NWIS donne un libre accès aux informations et mesures issues de la validation de terrain en plus de leur statut (mesures provisoires, invalidées, validés) quand la BH ne fournit qu'un indicateur de qualité (mesures provisoires, invalidées, validés 'douteux' et validés 'bons').

\begin{tabular}{|l|l|}
\hline \multicolumn{1}{|c|}{$\begin{array}{c}\text { National Water Information } \\
\text { System (NWIS) }\end{array}$} & \multicolumn{1}{c|}{ Banque Hydro (BH) } \\
\hline $\begin{array}{l}\text { Présentation de la station } \\
\text { hydrométrique }\end{array}$ & $\begin{array}{l}\text { Présentation de la station } \\
\text { hydrométrique [STATION] }\end{array}$ \\
\hline Géolocalisation (WebGIS et carte) & $\begin{array}{l}\text { Coordonnées géographiques et carte } \\
\text { (pas de WebGIS) }\end{array}$ \\
\hline $\begin{array}{l}\text { Extraction spatiotemporelle ou } \\
\text { sémantique }\end{array}$ & $\begin{array}{l}\text { Extraction spatiotemporelle ou } \\
\text { sémantique }\end{array}$ \\
\hline $\begin{array}{l}\text { Hauteurs instantannées, jour, mois, } \\
\text { année }\end{array}$ & $\begin{array}{l}\text { Hauteurs instantannées, jour, mois, } \\
\text { année [H-TEMPS] }\end{array}$ \\
\hline Débits instantannés, jour, mois, année & $\begin{array}{l}\text { Débits instantannés, jour, mois, année } \\
\text { [QJM] }\end{array}$ \\
\hline Données historiques (19 ${ }^{\mathrm{e}}$-20 $0^{\mathrm{e}}$ siècles) & Données historiques (20 siècle) \\
\hline $\begin{array}{l}\text { Statistiques journalières, mensuelles, } \\
\text { annuelles }\end{array}$ & $\begin{array}{l}\text { Etude statistique des débits de crue } \\
\text { [CRUCAL] }\end{array}$ \\
\hline Valeurs extrêmes & $\begin{array}{l}\text { Débits maximaux/minimaux, étude } \\
\text { statistique [VCX-QCX/VCN-QCN] }\end{array}$ \\
\hline $\begin{array}{l}\text { Validation terrain et qualité de la } \\
\text { donnée }\end{array}$ & Validation et qualité de la donnée \\
\hline $\begin{array}{l}\text { Visualisation graphique des } \\
\text { chroniques }\end{array}$ & $\begin{array}{l}\text { Débits à pas de temps fixe/variable } \\
\text { [QTFIX/QTVAR] }\end{array}$ \\
\hline Synthèse hydrométrique annuelle & $\begin{array}{l}\text { Synthèse hydrométrique } \\
\text { [SYNTHESE] }\end{array}$ \\
\hline
\end{tabular}

Tableau 2. Les IDG en hydrométrie : le National Water Information System (USA) et la Banque Hydro (France).

Les IDG en imagerie et celles en hydrométrie n'offrent pas à l'utilisateur final un accès aux données basée sur la dynamique spatiotemporelle de leur observation mais uniquement à leur catalogage à partir des métadonnées. Ces dernières pourraient être enrichies pour proposer une interopérabilité « de niveau 2 »sur des méta-métadonnées (Willard et Berbert, 1999) en croisant les informations des métadonnées avec des objets spatiotemporel extraits des données de plusieurs IDG 
(Wiemann et Bernard, 2016, Willard et Berbert, 1999), dans le cas présent, les observatoires hydrométriques et l'imagerie aérienne ou satellitaire. S'agissant des volumes de données considérables déjà archivés et à venir, il est raisonnable de considérer cette nouvelle interopérabilité comme un défi numérique visant à proposer des métadonnées améliorées, orientées objet, sur les données ouvertes.

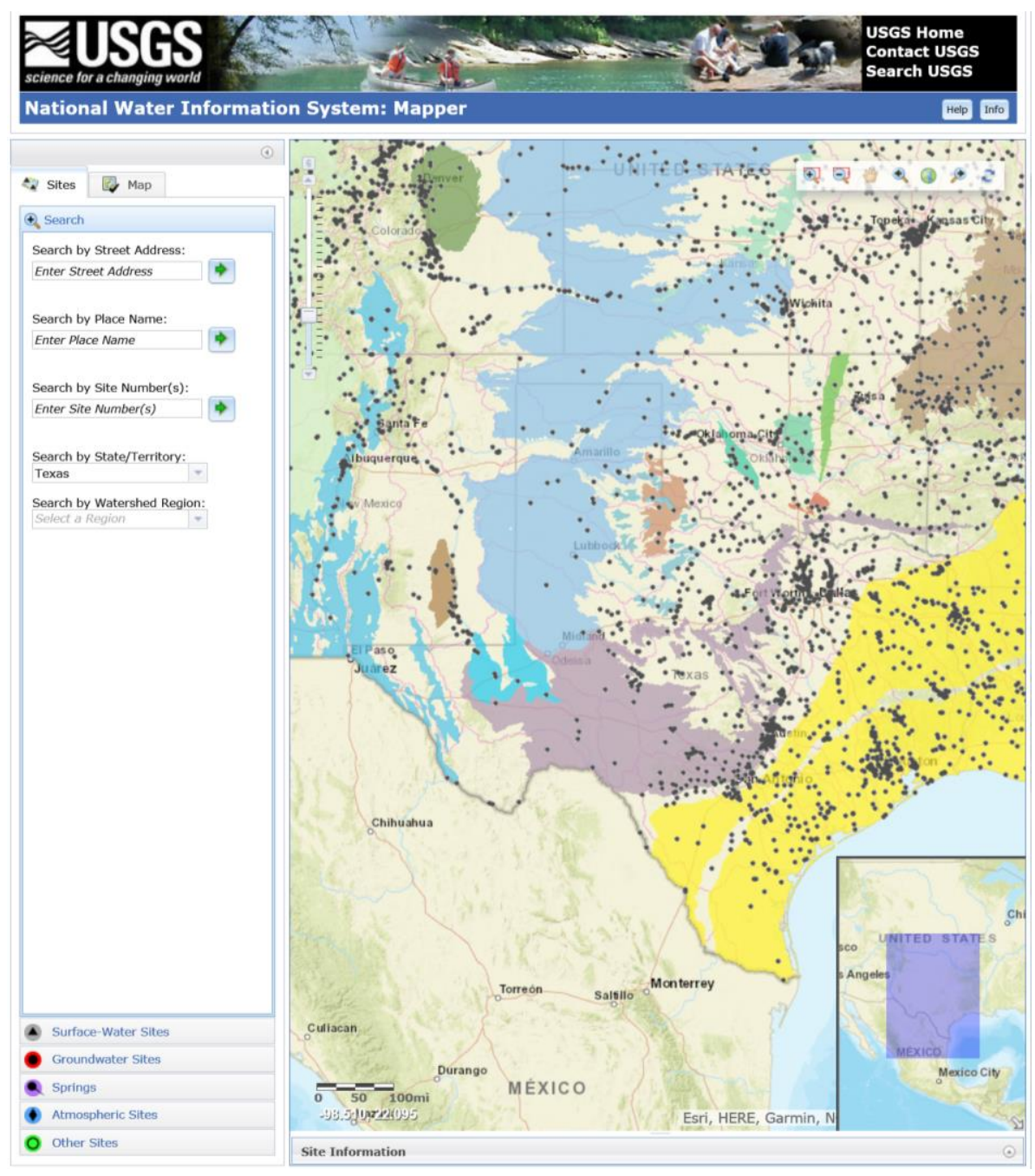

Figure 4. IDG du NWIS de I'USGS (https://waterdata.usgs.gov/nwis)

\section{Les défis numériques de l'observation spatio-temporelle des risques hydrologiques}

Les interfaces web des IDG n'implémentent pas la totalité des ressources documentaires contenues dans les métadonnées. Certains IDG restreignent le paramétrage des outils de requêtage spatiotemporel et attributaires aux informations les plus usuelles. Celles-ci ne permettent pas d'optimiser l'extraction de la donnée utile. Nous allons démontrer comment cette structuration est mal adaptée à la question des risques hydrologiques tant pour les données issues de l'imagerie que pour celles issues de l'hydrométrie.

\subsection{Dynamiques environnementales et évolutions technologiques de l'observation}

Nous abordons ici la question des dynamiques environnementales à partir de l'exemple des risques hydrologiques car ils sont à la fois très complexes à observer et à modéliser dans leurs dimensions spatiotemporelles.

L'acquisition de données de risque hydrologique par imagerie est limitée par la dynamique environnementale que l'on peut considérer ici comme l'évolution spatiotemporelle du contexte géographique dont la combinaison de processus conduit à l'émergence d'un événement hydrologique comme celui d'une inondation. Certaines crues sont lentes (crues des grandes plaines 
fluviales) d'autres crues, sont très rapides (crues torrentielles). Bien d'autres types de crues sont observables et leur dynamique temporelle est une réponse à la dynamique environnementale combinée de tous les processus participant au débordement des cours d'eau. Bien que les satellites à orbite polaire soient en mesure de faire le tour de la planète en une centaine de minutes, les crues rapides sont des phénomènes dont l'intensité maximale est très fugace. Les inondations qui en résultent concernent des surfaces souvent géographiquement restreintes où la très haute résolution spatiale est nécessaire pour bien renseigner l'emprise géographique de la zone de submersion. Or l'imagerie à très haute résolution ne dispose pas de longues chroniques d'observation. Il y a donc peu d'événements historiques qui sont archivés à très haute résolution spatiale. S'agissant des crues lentes, la propagation du pic de crue d'amont en aval, qui s'accompagne d'une pulsation de débordement, est un phénomène qui ne peut pas s'observer avec précision en une seule image ni même dans une série d'images dont la fréquence de revisite dans des conditions d'observation similaires est au minimum de un à plusieurs jours (Kaur et al., 2019, Sichangi et al., 2011). L'observation de la propagation de l'extension maximale d'une inondation par imagerie est donc aléatoire et opportune, même sans tenir compte des conditions atmosphériques qui peuvent encore limiter l'accès «théorique » du capteur à l'état de surface au sol (Jain et al., 2005 ; Hoque et al. 2011). L'observation par imagerie est difficile à synchroniser avec l'objet spatiotemporel « inondation ».

La connaissance de l'aléa hydrologique et sa comparaison avec des épisodes d'inondations antérieurs nécessitent l'accès à de longues chroniques hydrométriques pour lesquelles les conditions de stationnarité d'observation doivent être respectées. Nous avons déjà évoqué la difficulté de maintenir ce critère de robustesse de la mesure hydrométrique car de multiples facteurs concourent à dégrader les conditions et protocoles d'observations. L'évolution des conditions d'écoulement dans le lit mineur (phénomènes d'érosion/sédimentation, entretien des rivières) influencent le déclenchement de l'inondation. Dans le lit majeur, l'évolution de l'occupation du sol modifie également les conditions d'écoulement. A l'échelle d'un événement, les hydrologues observent une hystérésis entre la phase de crue et celle de la décrue. Les mesures hydrométriques ne restituent donc pas rigoureusement l'ensemble de la dynamique temporelle de l'évolution des écoulements bien que la mesure des hauteurs d'eaux soit paradoxalement très fiable. Là encore le système d'observation du risque hydrologique restitue une "réalité approchée » par un modèle local d'écoulement: la courbe de tarage. L’objet «aléa hydrologique » nécessite une observation temporelle complexe dans laquelle les états les écoulements observés sont sous la dépendance des conditions hydrométriques précédentes. De ce fait la durée d'un épisode d'inondation est très variable. Dans le cas d'une acquisition de mesure hydrométrique à haute fréquence, il est utile de pouvoir identifier/extraire le temps de base d'un épisode d'inondation afin de pouvoir restreindre l'extraction de données hydrométriques utiles pour caractériser l'événement hydrologique par différents paramètres (temps de montée, temps de base), éventuellement croisés avec des données pluviométriques (temps de réponse et temps de concentration). Cela revient à réaliser une segmentation dans la succession temporelle des données hydrométriques (Gu et al., 2016; Kundzewicz et al., 2005 ; Nied et al., 2014 ; Svensson et al., 2005 ; Villarini et al. 2013 ; Wang et $a l ., 2015)$ pour délimiter un objet numérique équivalent à un événement, une « fenêtre temporelle », correspondant également au signal hydrologique de l'objet spatiotemporel «crue/inondation » (Singh et al., 2011).

L'aléa hydrologique est donc un objet complexe qui peut s'extraire par segmentation de données spatiotemporelles (imagerie et chroniques hydrométriques). Plus intéressant pour l'utilisateur final, serait l'extraction d'un objet numérique dont la segmentation s'appuierait sur plusieurs IDG (Wiemann et Bernard, 2016) et sur une interopérabilité à partir de métadonnées enrichies. Or actuellement, l'observation numérique d'un objet spatiotemporel tel qu'une inondation est distribuée sur plusieurs IDG qui utilisent un MLD de catalogage en silo. 


\subsection{Big data, métadonnées et interopérabilité}

Le stockage des big data géospatiales et hydrométriques ouvert et interopérable constitue un volume de données au sein duquel des objets peuvent être extraits, voir segmentés, pour chaque jeu de donnée (Wiemann et Bernard, 2016 ; Villarini et al., 2013 ; Sichangi et al. 2011 ; Percivall, 2010 ; Nied et al. 2014 ; Hoque et al., 2011 ; Demir et Krajewski, 2013, Dao et Liou, 2015 ; Bernard et al., 2017, Ames et al., 2012). Cependant 1'utilisateur final, hydrologue géographe ou télédétecteur, acteur public ou acteur privé recherche surtout à croiser toutes ces informations pour caractériser au mieux un événement hydrologique et son emprise spatiale. Pour y arriver, il fait, pour toutes les données qu'il juge utiles, autant d'extractions dans autant d'IDG auxquelles il peut accéder. Puis, il construit/alimente une base de données géographique au sein de laquelle il va expertiser l'intégralité de la donnée collectée pour confronter les critères de sélection sur métadonnées aux critères d'utilisation opérationnelle dans ses propres chaînes de traitement. Par exemple, il télécharge une image satellitaire à $10 \%$ de couverture nuageuse car c'est la seule disponible sur la fenêtre temporelle qu'il recherche pour des paramètres instrumentaux définis (résolutions spectrales, spatiales et numériques). Dans l'IDG, l'aperçu sur cette donnée satellitaire n'offre pas une lisibilité suffisante, ce qui a d'ailleurs motivé le téléchargement de l'image entière afin de faire un contrôle utilisateur avec l'image à pleine résolution. Dans cette dernière phase de contrôle utilisateur, il aurait souhaité faire une requête sur la couverture nuageuse qui tienne compte de sa zone d'intérêt. Malheureusement la couverture nuageuse est calculée à l'échelle de l'image entière. La probabilité que la zone d'intérêt de l'utilisateur soit sans nuage est non nulle. Le système de requêtage d'un IDG ne permet pas non plus de savoir si, dans une image satellitaire à $90 \%$ de couverture nuageuse, la probabilité d'observer une zone d'intérêt utilisateur est nulle. Si dans un premier IDG, aucune image satellitaire n'offre une acquisition qui correspond à l'objet spatiotemporel étudié, qu'en est-il des autres producteurs de données ? Quelle sera le seuil de couverture nuageuse qui permettra d'identifier la fenêtre atmosphérique qui croisera l'emprise spatiale de la zone géographique d'intérêt pour l'utilisateur? Si l'utilisateur s'oriente vers une image radar pour éliminer le problème de nébulosité atmosphérique pourra-t-il disposer d'une métadonnée de vitesse de vent en surface pour garantir une détection optimale de la surface inondée ? Quelle que soit l'imagerie choisie, y aura-t-il une station hydrométrique disposant d'un suivi complet de l'événement hydrologique dans la zone d'intérêt ? Si oui, disposera-t-elle de chroniques suffisantes et de bonne qualité pour calculer des indicateurs hydrométriques classiques (période de retour, courbe IDF : Intensité-Durée-Fréquence) ? Dans la zone couverte par l'imagerie ? Répondre à ces questions implique une recherche fédérée mobilisant plusieurs IDG dont certains regroupent pourtant plusieurs catalogues (Earthexplorer). Il faut également que les métadonnées fournies à l'échelle de l'image soient mises à jour et enrichies car, dans les IDG actuelles, la donnée d'imagerie est sous-renseignée, parce que la métadonnée est uniquement produite à l'échelle globale de l'image (Desconnets, 2017, Willard et Berbert, 1999). Mettre en œuvre une segmentation robuste de type quadtree (Finkel et Bentley, 1974 ; Bentley, 1975 ; Samet, 1984) puis extraire de nouvelles métadonnées dans une couche vectorielle interopérable permettrait de résoudre la partie spatiale du problème de l'utilisateur travaillant sur les aléas hydrologiques. Il est en effet assez facile de recalculer des métadonnées enrichies pour chaque objet segmenté en lieu et place d'une métadonnée globale à l'échelle de l'image. Pour les données Sentinel, l'ESA implémente la directive européenne INSPIRE et propose des données au format XFDU (XML Formated Data Unit), qui incluent des métadonnées vectorielles auxiliaires (figure 5) dont certaines, si elles étaient au format vectoriel, pourraient être utilisées pour optimiser la recherche de donnée utile.

Du côté de l'hydrométrie, l'information ponctuelle/temporelle est géolocalisée. Il est donc possible d'interroger la position géographique mais aussi la position temporelle de la mesure en rivière. Par contre comme pour les données images, il manque une segmentation temporelle des événements hydrologiques qui permettrait de n'extraire que l'information utile (Wang et al., 2015 ; Villarini et al., 2013 ; Merz et al., 2016, 2018 ; Gu et al., 2016 ; Bormann et al. 2011) voir même de définir une fenêtre temporelle pour alimenter une requête dans un autre IDG. Si extraire un signal de 
crue avec débordement est assez aisé, il est en revanche plus difficile d'extraire l'ensemble des signaux hydrologiques en lien avec un épisode de crue. En effet, en hydrologie il y a une interdépendance entre un débit observé et ceux qui le précèdent ou qui lui succèdent. Il existe donc des signaux numériques dans les données qui doivent être associés à l'analyse de l'événement observé. Cette sensibilité aux conditions antérieures (conditions initiales) est un élément important pour l'analyse d'un événement hydrologique. En croisant sur un espace utilisateur des données temporelles d'observation du couvert nuageux (IDG satellitaire) et des chroniques hydrométriques en rivière (IDG hydrologique), il est déjà plus facile de segmenter une fenêtre temporelle d'intérêt pour l'analyse des événements hydrologiques extrêmes.

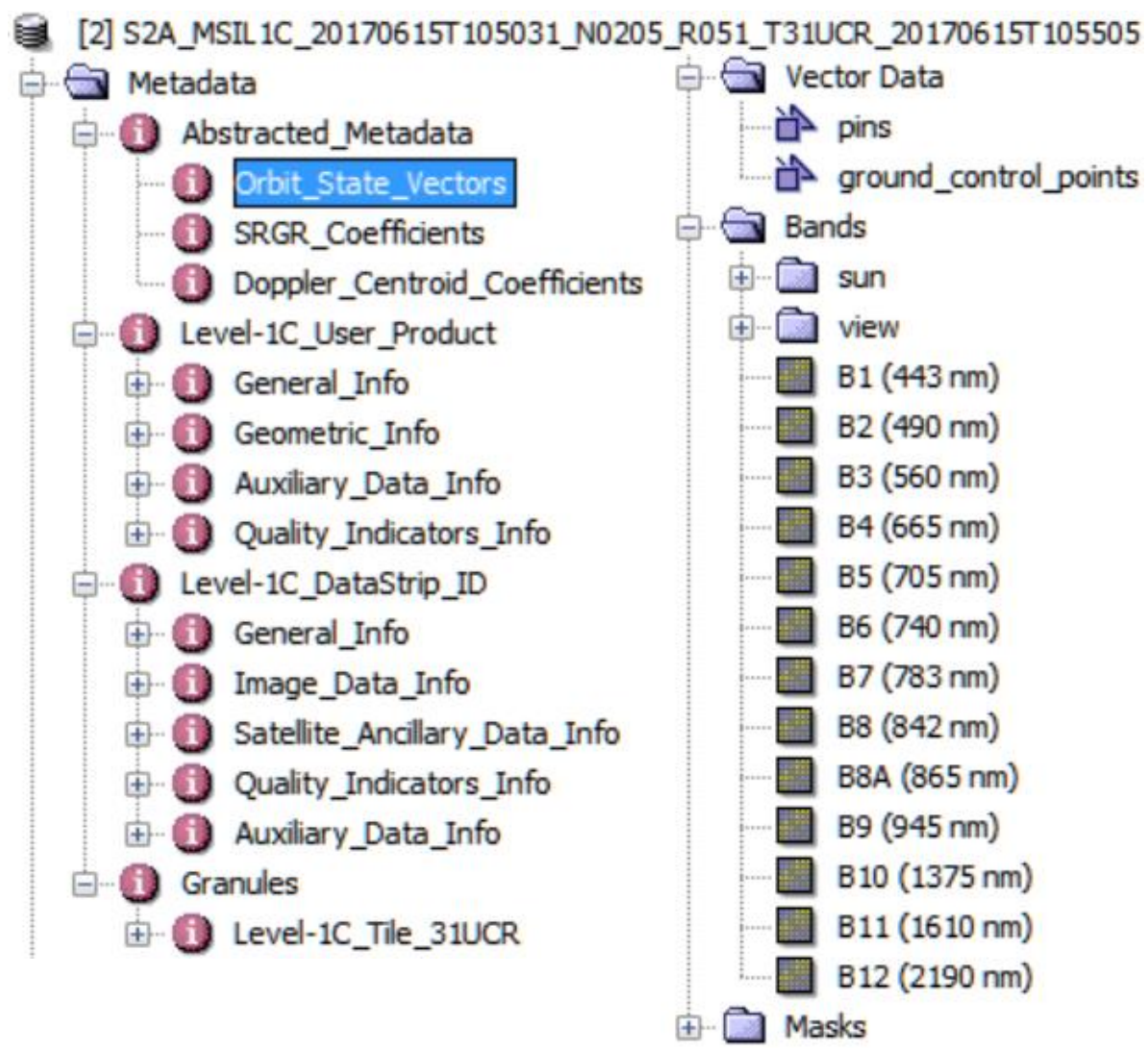

Figure 5. Visualisation des métadonnées d'une archive Sentinel 2A dans le logiciel SNAP de l'ESA (la dernière catégorie Masks contient 23 types de métadonnées dont une extraction des nuages au format image non requêtable).

Ces deux types de segmentation spatiale et temporelle utilisent des d'outils et des modèles de traitements qui sont faciles à mettre en œuvre. Ils peuvent donc aisément participer à la création d'une métadonnée améliorée (Willard et Berbert, 1999) qui permettrait de proposer à l'utilisateur une interopérabilité de niveau supérieur entre les IDG. Un aléa hydrologique, une fois observé par imagerie et par hydrométrie, disposerait alors d'une signature numérique inter IDG. Cette signature aurait une emprise spatiotemporelle numérique interopérable, accompagnée d'un jeu de métadonnées orientées objet. Cette emprise spatiotemporelle serait alors, dans l'espace numérique inter IDG, l'avatar numérique de l'objet hydrologique observé dans le «monde réel ». L'objet numérique serait aussi doté d'une dynamique numérique dont la signature s'exprime dans un système numérique produit par la mise en relation de différents jeux de données d'observation du monde réel. Ce système numérique (figure 6) peut être dimensionné selon une granularité :

- - spatiale : maille de la segmentation réalisée dans l'image,

- - numérique : profondeur d'encodage de 1 bits à 16 bits et plus selon la précision de la mesure),

- - paramétrique : spectrale pour l'imagerie, hauteur/volume/durée pour l'hydrométrie, 
- - temporelle: activité mesurable d'une signature numérique entre plusieurs observations enregistrées dans différentes IDG.

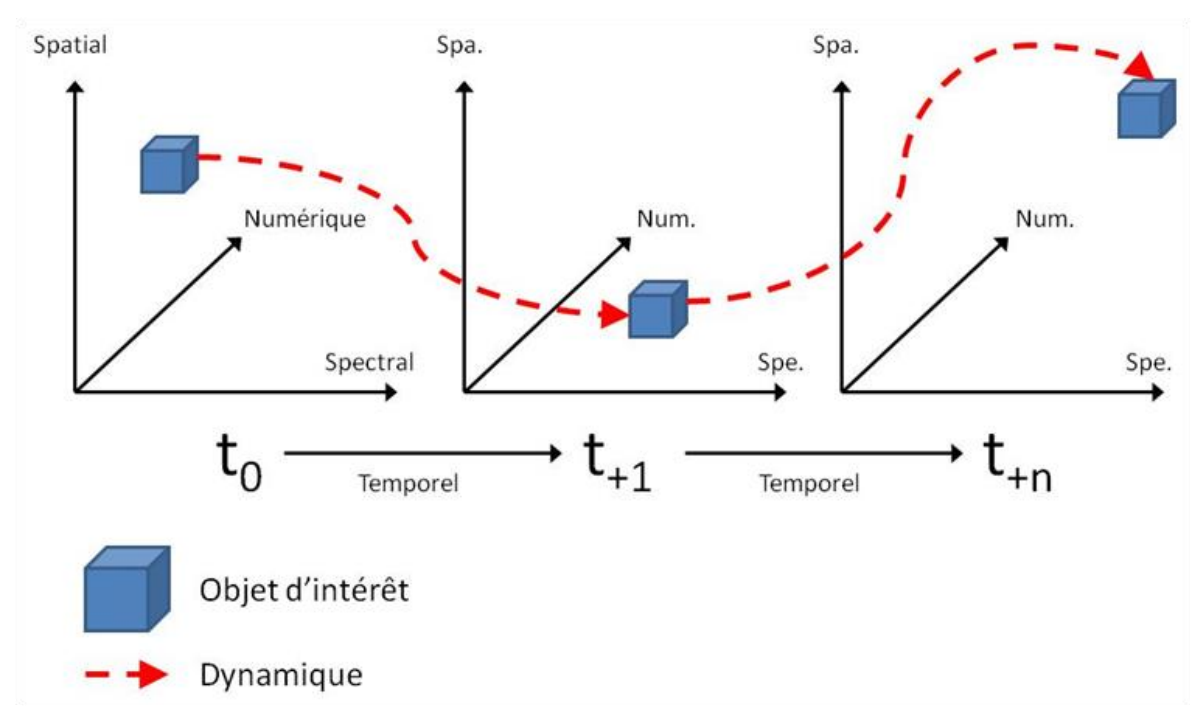

Figure 6. Trajectoires numériques des objets spatiotemporels dynamiques.

Produire une fouille de données dans ce contexte numérique quadridimensionnel revient alors à segmenter les big data pour y extraire des signatures numériques d'un ou plusieurs objet(s) observé(s) du monde réel à partir de plusieurs producteurs de données numériques. Le caractère dynamique d'un aléa hydrologique dans le monde physique réel aurait également un réciproque dans les big data sous la forme d'un objet dont la signature numérique permettrait d'identifier et de suivre la trajectoire numérique de cet objet par ses traces observables/«fouillables » dans un « écosystème numérique » d'IDG interopérables. Il y a pour y parvenir plusieurs défis numériques à relever.

\section{Conclusions et perspectives}

Dans cette conclusion nous présentons quelques pistes de réflexions sur ce que peuvent êtres les défis numériques en lien avec les questionnements abordés dans cet article. Elles sont proposées en prenant la posture de l'utilisateur final des données, celui qui veut optimiser son effort de collecte, de validation croisée et de suivi spatiotemporel des objets du monde réel qui l'intéressent en s'appuyant sur les données ouvertes fournies par les IDG. Il cherche ainsi à capitaliser l'information contenue dans les multiples observations disponibles pour répondre aux objectifs d'observatoire des dynamiques environnementales. Ces défis recoupent pour une part ceux qui ont été identifiés par J.C. Desconnets (2017, p. 44) : «Malgré ces efforts de standardisation, l'efficacité et la précision des recherches sur de grands ensemble de données spatiales... demeurent encore un grand défi à relever.». Il identifie notamment trois grands verrous (Desconnets, 2017): une ambiguïté sémantique dans les métadonnées (variables d'un fournisseur à l'autre), des modèles de recherche limités (opérateurs boléens et recherche de plein texte) et les IMH, interfaces homme machine (requéteurs), qui permettent à l'utilisateur de dialoguer avec et de sélectionner dans l'archivage des données via une exploration restreinte aux métadonnées imposées par le producteur/fournisseur.

Un premier défi numérique serait déjà de proposer des outils d'interrogation adaptatifs en fonction d'un contenu de métadonnées, elles-mêmes variables selon les producteurs, avec la possibilité d'interroger tous les critères disponibles dans la perspective proposée par Percivall (2010), Ames et al. (2012), Bernard et al. (2017), Demir et Krajewski (2013), Noucher et al. (2017) et Tarboton et al. (2009). Il s'agit ici de mettre en œuvre une recherche fédérée sur des IDG qui ne le sont pas forcément. L'utilisateur n'utiliserait plus l'IMH par défaut de chaque IDG mais un moteur de recherche fédérée. Ce moteur devrait pouvoir proposer un classement et un score des 
réponses en fonction des éléments d'une requête classique (espace, temporalité, qualité) mais aussi des caractéristiques paramétriques pour les observations alternatives proposées (bandes spectrales échantillonnées, résolution spatiale, outil de mesure de la hauteur d'eau...). Cette approche renvoie aux outils de recherche de type Relevance Feedback (Costache, Maitre et Datcu, 2006 ; Salton et Buckley, 1990) et implique le respect des recommandations et protocoles d'interopérabilité promus par le groupe W3C/OGC (van den Brink et al., 2019; Haller et al., 2018). Ces outils devraient pouvoir interroger des métadonnées interopérables sur plusieurs IDG mais aussi pouvoir fédérer des métadonnées relevant de plusieurs silos d'une même IDG. Ce premier défi renvoie aux concepts de méta-métadonnées (Willard et Berbert, 1999) et ceux promus par l'ISDE (International Society for Digital Earth) qui insiste sur le fait que des questions scientifiques complexes ne peuvent pas être résolues à l'intérieur d'un seul domaine scientifique et que les digital earth technologies doivent pouvoir répondre aux besoins d'échanges, d'intégration et de réemploi de données hétérogènes (Janowicz et Hitzler, 2012).

Un deuxième défi numérique serait de tenir compte de la dérive technologique, instrumentale dans les longues chroniques d'observation ou leurs ontologies (Schlieder, 2010) en proposant là encore des indicateurs de qualité (Devillers et al., 2007 ; Desconnets, 2017), d'équivalence, de reconstruction de lacunes (données Landsat 7 postérieures à 2003), de dégradation/alignement des données les plus récentes sur les données plus anciennes (figure 1). Cela reviendrait à proposer un post-traitement de la donnée brute à partir d'un catalogue de données réparties sur plusieurs IDG. L'utilisateur trouverait dans ce type d'outil un moyen de convertir les données disponibles en information numérique utile aux objectifs recherchés. Là encore il s'agit de s'affranchir de la structuration de la donnée en silos qui reste un verrou majeur dans l'exploitation des données accumulées dans les IDG. Aussi ambitieux que cela puisse paraitre, ce deuxième défi est au cœur de la proposition Earth Engine de Google dont l'ambition est clairement affichée sur sa page d'accueil «Google Earth Engine associe un catalogue d'imagerie par satellite et d'ensembles de données géospatiales de plusieurs pétaoctets à des fonctionnalités d'analyse à l'échelle planétaire. Il est également mis à la disposition des scientifiques, des chercheurs et des développeurs pour détecter les changements, cartographier les tendances et quantifier les différences à la surface de la Terre » (https://earthengine.google.com/). C'est aujourd'hui la proposition qui se rapproche le plus des objectifs de ce second défi pour la partie imagerie spatiale et aéroportée.

Un troisième défi consisterait à produire et à utiliser une métadonnée de «deuxième niveau » (Willard et Berbert, 1999) qui ne soit plus seulement une métadonnée globale à l'échelle de l'observation ou de sa chronique mais une métadonnée de niveau supérieur issue d'un corpus d'indicateurs produit par post-traitement sur les données brutes et permettant d'extraire :

- - un objet d'intérêt géographique : par segmentation spatiale des données à l'intérieur d'une image en mobilisant des informations exogènes (utilisateur),

- - un objet d'intérêt temporel : par segmentation d'événements dans les chroniques ou séries temporelles des observations (imagerie et chroniques hydrométriques).

- - un objet numérique spatio-temporel (figure 6) : par segmentation de la trace numérique qu'il peut laisser dans différentes IDG (une inondation observée par plusieurs plateformes satellitaires et plusieurs stations de mesure hydrométriques).

Ce troisième deuxième défi relève de l'extraction d'objets numériques spatiotemporels à partir des données archivées pour mieux renseigner la métadonnée et l'utilisateur sur la valeur thématique d'usage d'un enregistrement dans une IDG. Il s'agit là encore d'une proposition qui se positionne entre l'acquisition/production et l'utilisation/valorisation thématique de la donnée numérique. Bien qu'elle repose sur des traitements numériques bien maîtrisés, elle présente un coût de calcul non négligeable à l'échelle des big data géospatiales. En effet ce type d'extraction mobilise "des opérateurs topologiques (intersection, inclusion, visinage) dont la complexité de calcul est élevée. » (Desconnets, 2017, p. 68). Enfin, la question de la temporalité des objets numériques pose le 
problème du «Digital Heritage» (Schielder C., 2010) qui implique la prise en compte d'une ambiguité et d'une dérive sémantique temporelle dans les ontologies qui alimentent les métadonnées.

La donnée acquise par un observatoire est actuellement gérée et produite selon un MLD gouverné par les paramètres techniques de l'observation et par les moyens opérationnels et financiers pour faire fonctionner les IDG (Chevalier et Caron, 2002). Cette gouvernance très verticale (Raj Budhathoki et al., 2008. Noucher et Gautreau, 2013 ; Georis-Creuseveau et al. 2015) conduit à une juxtaposition de silos de données alors que l'utilisateur final souhaiterait disposer d'un outil de collecte transversal qui réponde à ces objectifs de terrain et de suivi des dynamiques environnementales (da Silva et Camboim, 2018 ; Noucher et Gautreau, 2014). Ce repositionnement de l'utilisateur final sur la création, l'usage et l'interopérabilité des métadonnées, éventuellement enrichies croise les objectifs, les enjeux et les nouvelles technologies du web sémantique appliqué aux données spatiales (Haller et al., 2019 ; van den Brink et al., 2019).

Laisser les utilisateurs finaux définir seuls des protocoles d'acquisition/observation et de gestion des IDG n'est pas une solution. Cela reviendrait à faire de l'observation «sur-mesure », au détriment des objectifs globaux des observatoires EO, des objectifs de stationnarité des observatoires hydrologiques et des objectifs de continuité temporelle de toute observation nécessaire au suivi dynamique de l'environnement. La granularité optimale doit donc se situer entre l'offre des fournisseurs/producteurs d'observations et les demandes des chercheurs/usagers des IDG. Cette recherche de compromis n'est pas un objectif nouveau (Salton et Buckley, 1990 ; Smith, 1996 ; Willard et Berbert, 1999) mais l'évolution technologique des capacités d'observation et l'évolution thématique des approches pluridisciplinaires des observatoires imposent une évolution dynamique des outils de recherche s'appuyant sur l'interopérabilité des données spatiales et sur les outils du web sémantique.

\section{Remerciements}

Les auteurs remerciement vivement les relecteurs anonymes dont les conseils et les remarques ont permis d'améliorer ce papier et d'en élargir les perspectives. Ils remercient également André Miralles (IRSTEA) pour ses recommandations apportées dans les premières versions du manuscrit avant soumission à la revue.

\section{Bibliographie}

Aditya T., Kraak M.-J. (2007). A Search Interface for an SDI: Implementation and Evaluation of Metadata Visualization Strategies. Transactions in GIS, 2007, 11(3), pp. 413-435. DOI: https://doi.org/10.1111/j.14679671.2007.01053.x.

Ames D. P., Horsburgh J. S., Cao Y., Kadlec J., Whiteaker T., Valentine D. (2012) HydroDesktop: Web servicesbased software for hydrologic data discovery, download, visualization, and analysis. Environmental Modelling \& Software, Vol. 37, November 2012, pp. 146-156. DOI: https://doi.org/10.1016/j.envsoft.2012.03.013.

Bentley J. L. (1975). Multidimensional Binary Search Trees Used for Associative Searching. Communications of the ACM, September 1975, Vol. 18, No. 9, pp. 509-517. DOI: https://doi.org/10.1145/361002.361007.

van den Brink, B. and Piasecki M. (2009). Engineering new paths to water data. Computers \& Geosciences, 35(4): pp. 753-760. DOI: http://dx.doi.org/10.1016/j.cageo.2008.02.017.

Bernard C., Villanova-Oliver M., Gensel J., Le Rubrus B. (2017), Spatio-Temporal evolutive Data Infrastructure: a Spatial Data Infrastructure for managing data flows of Territorial Statistical Information, International Journal of Digital Earth, Volume 10, 2017 - Issue 3, pp. 257-283. DOI: https://doi.org/10.1080/17538947.2016.1222003.

Borah S. B., Sivasankar T., Ramya M. N. S., Raju P. L. N., (2018). Flood inundation mapping and monitoring in Kaziranga National Park, Assam using Sentinel-1 SAR data. Environ. Monit. Assess. 190 (9): 520. DOI: https://doi.org/10.1007/s10661-018-6893-y. 
Bormann H., Pinter N., Elfert S., (2011). Hydrological signatures of flood trends on German rivers: Flood frequencies, flood heights and specific stages. Journal of Hydrology, 404 (2011), pp. 50-66. DOI : http://dx.doi.org/10.1016/j.jhydrol.2011.04.019.

Chevalier J.-J. et Caron C. (2002). Développement d'Infrastructures Géomatiques : déterminisme technologique ou approche holistique. Symposium sur la théorie, les traitements et les applications des données Géospatiales 2002, Ottawa, 9 p.

Chevallier R. (1963). Panorama des applications de la photographie aérienne. In: Annales. Economies, sociétés, civilisations. 18e année, N. 4, 1963. pp. 677-698. DOI: https://doi.org/10.3406/ahess.1963.421036.

Chung H., Liu C., Cheng I., Lee Y., Shieh M. (2015). Rapid response to a typhoon-induced flood with an SARderived map of inundated areas: Case study and validation. Remote Sensing, 7(9), pp. 11954-11973. DOI: https://doi.org/10.3390/rs70911954.

Costache M., Maitre H., Datcu M. (2006). Categorization based Relevance Feedback Search Engine for Earth Observation Images Repositories. IEEE International Symposium on Geoscience and Remote Sensing, 31 July-4 Aug. 2006, Denver (CO, USA), 4 p. DOI: https://doi.org/10.1109/IGARSS.2006.8.

da Silva E. S., Camboim S. P. (2018). Eliciting academic SDI requirements through a survey of user practices. Open Geospatial Data, Software and Standards (2018), 3:11, 10 p. DOI: https://doi.org/10.1186/s40965-018-0054-7.

Dao P. D., Liou Y.-A. (2015). Object-based flood mapping and affected rice field estimation with landsat 8 OLI and MODIS data. Remote Sensing, 7(5), pp. 5077-5097. DOI: https://doi.org/10.3390/rs70505077.

Desconnets J.-C. (2017). Recherche d'information spatio-temporelle : Application aux images satellitaires. Habilitation à Diriger des Recherches, Spécialité: Informatique, soutenue le 17 novembre 2017, Maison de la Télédétection, Montpellier, 143 p. https://hal.archives-ouvertes.fr/tel-01649173v2/document

Demir I., Krajewski W. F. (2013). Towards an integrated Flood Information System: Centralized data access, analysis, and visualization. Environmental Modelling and Software, 50, pp. 77-84. DOI: https://doi.org/10.1016/j.envsoft.2013.08.009.

Devillers R., Bédard Y., Jeansoulin R., Moulin B. (2007). Towards spatial data quality information analysis tools for experts assessing the fitness for use of spatial data, International Journal of Geographical Information Science, 21:3, pp. 261-282, DOI: http://dx.doi.org/10.1080/13658810600911879.

Finkel R. A., Bentley J. L., (1974) Quad Trees A Data Structure for Retrieval on Composite Keys. Acta Informatica 4, pp. 1-9. DOI: https://doi.org/10.1007/BF00288933.

Frazier P., Page K., Louis J., Briggs S., Robertson A. I. (2003). Relating wetland inundation to river flow using Landsat TM data. International Journal of Remote Sensing, 24(19), pp. 3755-3770. DOI: https://doi.org/10.1080/0143116021000023916.

Georis-Creuseveau J. (2014). Les Infrastructures de Données Géographiques (IDG) : développement d'une méthodologie pour l'étude des usages. Le cas des acteurs côtiers et de la GIZC en France. Thèse de doctorat de Géographie, Université de Bretagne Occidentale, 281 p.

Georis-Creuseveau J., Gourmelon F., Claramunt C., (2015). Infrastructures de données géographiques : quelle contribution à la Gestion intégrée des zones côtières françaises ? », VertigO - la revue électronique en sciences de l'environnement [En ligne], Volume 15 Numéro 1, mai 2015, http://journals.openedition.org/vertigo/15857

Gu X., Zhang Q., Singh V. P., Chen Y. D., Shi P., (2016).Temporal clustering of floods and impacts of climate indices in the Tarim River basin, China. Global and Planetary Change, 147 (2016), pp. 12-24. DOI: http://dx.doi.org/10.1016/j.gloplacha.2016.10.011.

Haller A., Janowicz K., Cox S.-J. D., Lefrançois M., Taylor K., Le Phuoc D., Lieberman J., García-Castro R., Atkinson R., Stadler C. (2019). The modular SSN ontology: A joint W3C and OGC standard specifying the semantics of sensors, observations, sampling, and actuation. Semantic Web, vol. 10, no. 1, pp. 9-32. DOI: https://doi.org/10.3233/SW-180320.

Hoque R., Nakayama D., Matsuyama H., Matsumoto J. (2011). Flood monitoring, mapping and assessing capabilities using RADARSAT remote sensing, GIS and ground data for Bangladesh. Natural Hazards, 57(2), pp. 525-548. DOI: https://doi.org/10.1007/s11069-010-9638-y.

Jain S. K., Singh R. D., Jain M. K., Lohani A. K. (2005). Delineation of flood-prone areas using remote sensing techniques. Water Resources Management, 19(4), pp. 333-347. DOI: https://doi.org/10.1007/s11269-005-3281-5.

Janowicz K. (2010). The role of space and time for knowledge organization on the Semantic Web. Semantic Web, vol. 1, no. 1, 2, pp. 25-32. DOI: https://doi.org/10.3233/SW-2010-0001. 
Janowicz K., Hitzler P. (2012). The Digital Earth as knowledge engine. Semantic Web, vol. 3, no. 3, pp. 213-221. DOI: https://doi.org/10.3233/SW-2012-0070.

Kharroubi O. (2013). Prévision des crues par modèle de réseau de neurones artificiels : application au bassin versant de l'Eure. Thèse de doctorat d'Aménagement et d'Urbanisme, Université Lille 1 Sciences et Technologies, 211 p.

Kharroubi O., Blanpain O., Masson E., Lallahem S. (2016). Application of artificial neural networks to predict hourly flows: case study of the Eure basin, France, Hydrological Sciences Journal, 61:3, 541-550, DOI: https://doi.org/10.1080/02626667.2014.933225.

Kaur A., Ghawana T., Kumar N. (2019) Preliminary Analysis of Flood Disaster 2017 in Bihar and Mitigation Measures. In: Rao P., Rao K., Kubo S. (eds) Proceedings of International Conference on Remote Sensing for Disaster Management. Springer Series in Geomechanics and Geoengineering. Springer, Cham, DOI https://doi.org/10.1007/978-3-319-77276-9_40.

Khan S. I., Hong Y., Wang J., Yilmaz K. K., Gourley J. J., Adler R. F., Brakenridge G. R., Policelli F., Habib S., Irwin D. (2011). Satellite remote sensing and hydrologic modeling for flood inundation mapping in lake victoria basin: Implications for hydrologic prediction in ungauged basins. IEEE Transactions on Geoscience and Remote Sensing, 49(1 PART 1), pp. 85-95. DOI: http://doi.org/10.1109/TGRS.2010.2057513.

Knebl, M. R., Yang Z., Hutchison K., Maidment D. R. (2005). Regional scale flood modeling using NEXRAD rainfall, GIS, and HEC-HMS/ RAS: A case study for the San Antonio River Basin Summer 2002 storm event. Journal of environmental management, $75(4 \quad$ SPEC. $\quad$ ISS.), pp. 325-336. DOI: https://doi.org/10.1016/j.jenvman.2004.11.024.

Kundzewicz Z. W., Graczyk D., Maurer T., Pińskwar I., Radziejewski M., Svensson C., Szwed M., (2005). Trend detection in river flow series: 1. Annual maximum flow / Détection de tendance dans des séries de débit fluvial: 1. Débit maximum annuel, Hydrological Sciences Journal, 50:5, pp. 797-810, DOI: http://dx.doi.org/10.1623/hysj.2005.50.5.797.

Masson E. (2002) Les conséquences des crues dans le bassin versant de l'Eure : Le risque inondation dans un hydrosystème anthropisé. Thèse de doctorat de Géographie, Université Caen Basse Normandie, 2 vol., 700 p.

Merz B., Nguyen V. D., Vorogushyn S., Temporal clustering of floods in Germany: Do flood-rich and flood-poor periods exist? Journal of Hydrology, 541 (2016), pp. 824-838. http://dx.doi.org/10.1016/j.jhydrol.2016.07.041.

Merz B., Viet Dung N., Apel H., Gerlitz L., Schröter K., Steirou E., Vorogushyn S. (2018). Spatial coherence of floodrich and flood-poor periods across Germany. Journal of Hydrology, 559 (2018), pp. 813-826. https://doi.org/10.1016/j.jhydrol.2018.02.082.

Nied M., Pardowitz T., Nissen K., Ulbrich U., Hundecha Y., Merz B., (2014). On the relationship between hydrometeorological patterns and flood types. Journal of Hydrology, 519 (2014), pp. 3249-3262. http://dx.doi.org/10.1016/j.jhydrol.2014.09.089.

Noucher M., (2016). Infrastructures de données géographiques et flux d'information environnementale. Netcom [En ligne], 27-1/2, 2013, 120-147. DOI : http://netcom.revues.org/1404

Noucher M., Gautreau P. (2014) Les données géographiques comme révélateur de l'état des connaissances sur l'environnement? Analyse et comparaison des infrastructures nationales brésilienne et française de données géographiques. Envibras 2014 : "Environnement et géomatique : approches comparées France - Brésil”, Nov 2014, Rennes, France. DOI : http://doi.org/10.13140/2.1.1308.6405

Noucher M., Gautreau P., (2013). Le libre accès rebat-il les cartes? Nouvelles perspectives pour les données géographiques. Les Cahiers du numérique, Lavoisier, 2013/1 Vol. 9, pp. 57-83. DOI: https://doi.org/10.3166/lcn.9.1.57-83.

Noucher M., Gourmelon F., Gautreau P., Georis-Creuseveau J., Maulpoix A., Pierson J., Pinède N., Pissoat O., Rouan M. (2017). Spatial Data Sharing: A Pilot Study of French SDIs. ISPRS International Journal of Geo-Information, 6, 99. DOI: https://doi.org/10.3390/ijgi6040099.

Percivall G. (2010). The application of open standards to enhance the interoperability of geoscience information, International Journal of Digital Earth, 3:S1, pp. 14-30, DOI: https://doi.org/10.1080/17538941003792751.

Raj Budhathoki N., Bruce B., Nedovic-Budic Z. (2008), Reconceptualizing the role of the user of spatial data infrastructure. GeoJournal, 72: 149-160. DOI : http://doi.org/10.1007/s10708-008-9189-x.

Revilla-Romero B., Hirpa F. A., Pozo J.T., Salamon P., Brakendridge R., Pappenberger F., de Gooeve T. (2015). On the use of global flood forecasts and satellite-derived inundation maps for flood monitoring in data-sparse regions. Remote Sensing, 7(11), pp. 15702-15728. DOI: https://doi.org/10.3390/rs71115702. 
Salton G., Buckley C. (1990). Improving retrieval performance by relevance feedback. Journal of the American Society for Information Science, Volume 41, Issue 4, June 1990, pp. 288-297.

Samet H. (1984). The Quadtree and Related Hierarchical Data Structures. Computing Surveys, Vol. 16, No. 2, June 1984, pp.187-260.

Schlieder C. (2010). Digital heritage: Semantic challenges of long-term preservation. Semantic Web, vol. 1, no. 1-2, pp. 143-147. DOI: https://doi.org/10.3233/SW-2010-0013.

Sichangi A. W., Wang L., Yang K., Chen D., Wang Z., Li X., Zhou J., Liu W. Kuria D., (2016). Estimating continental river basin discharges using multiple remote sensing data sets, Remote Sensing of Environment 179 (2016) 36-53. DOI: https://doi.org/10.1016/j.rse.2016.03.019.

Singh S. K., Pandey, A. C. and Nathawat M. S. (2011). Rainfall variability and spatio temporal dynamics of flood inundation during the 2008 Kosi flood in Bihar State, India. Asian Journal of Earth Sciences, Vol. 4, Iss. 1, pp. 9 19. DOI: http://dx.doi.org/10.3923/ajes.2011.9.19.

Smith T. R. (1996). A digital library for geographically referenced materials. Computer, Vol. 29, Issue. 5, pp. 54-60. DOI: http://dx.doi.org/10.1109/2.493457.

Souchon J.-P., Paparoditis N., Martin O., Meynard C., Thom C. (2006). Is There an Ideal Digital Aerial Camera ? Revue Française de Photogrammétrie et de Télédétection, nº 183, pp. 29-34.

Svensson C., Kundzewicz Z. W., Maurer T., (2005) Trend detection in river flow series: 2. Flood and low-flow index series / Détection de tendance dans des séries de débit fluvial: 2. Séries d'indices de crue et d'étiage, Hydrological Sciences Journal, 50:5, pp. 811-824, DOI: http://dx.doi.org/10.1623/hysj.2005.50.5.811.

Tarboton D.G., Horsburgh J.S., Maidment D.R., Whiteaker T., Zaslavsky I., Piasecki M., Goodall J., Valentine D., Whitenack T. (2009). Development of a Community Hydrologic Information System. $18^{\text {th }}$ World IMACS / MODSIM Congress, Cairns, Australia 13-17 July 2009, pp. 988-994.

Touzet T., Lecordix F. (2010). La carte forestière sans papier. CFC, N²06, pp. 53-62.

UNOSAT, 2017, Geospatial catalogue to support humanitarian operations, $2 \mathrm{p}$. https://www.unitar.org/unosat/sites/unitar.org.unosat/files/uploads/catalogue_factsheet_20072017_print.pdf

van den Brink L., Barnaghi P., Tandy J., Atemezing G., Atkinson R., Cochrane B., Fathy Y., García-Castro R., Haller A., Harth A., Janowicz K., Kolozali Ş., van Leeuwen B., Lefrançois M., Lieberman J., Perego A., Le-Phuoc D., Roberts B., Taylor K., Troncy R. (2019). Best practices for publishing, retrieving, and using spatial data on the web. Semantic Web, vol. 10, no. 1, pp. 95-114. DOI: http://doi.org/10.3233/SW-180305.

Vancauwenberghe G., Valekait K., van Loenen B., and Welle Donker F. (2018). Assessing the Openness of Spatial Data Infrastructures (SDI): Towards a Map of Open SDI. International Journal of Spatial Data Infrastructures Research, 13, 88-100. DOI: http://doi.org/10.2902/1725-0463.2018.13.art9

Villarini G., Smith J. A., Vitolo R., Stephenson D. B., (2013). On the temporal clustering of US floods and its relationship to climate teleconnection patterns. Int. J. Climatol. 33: 629-640. DOI: http://doi.org/10.1002/joc.3458.

Wang W., Chau K., Xu D.-M., Chen X.-Y. (2015). Improving Forecasting Accuracy of Annual Runoff Time Series Using ARIMA Based on EEMD Decomposition. Water Resour. Manag., Vol. 29, Iss. 8, pp. 2655-2675. DOI: https://doi.org/10.1007/s11269-015-0962-6.

Wang X., Xie H., (2018). A review on applications of remote sensing and geographic information systems (GIS) in water resources and flood risk management. Water, 2018, 10, 608; doi: https://doi.org/10.3390/w10050608.

Wiemann S., Bernard L. (2016) Spatial data fusion in Spatial Data Infrastructures using Linked Data, International Journal of Geographical Information Science, 30:4, pp. 613-636, DOI: https://doi.org/10.1080/13658816.2015.1084420.

Willard T., Berbert J. (1999). Finding coincident data from satellites: using "meta-metadata" to reduce load on archive. 16th IEEE Symposium on Mass Storage Systems in cooperation with the 7th NASA Goddard Conference on Mass Storage Systems and Technologies, 15-18 March 1999, DOI: http://10.1109/MASS.1999.830059. 